\title{
Entrelacs
}

Cinéma et audiovisuel

\section{Filmographie de Laurent Roth}

\section{(2) OpenEdition \\ 12 Journals}

Édition électronique

URL : https://journals.openedition.org/entrelacs/6202

DOI : 10.4000/entrelacs.6202

ISSN : 2261-5482

Éditeur

Éditions Téraèdre

Référence électronique

«Filmographie de Laurent Roth », Entrelacs [En ligne], 18| 2021, mis en ligne le 14 décembre 2021, consulté le 15 décembre 2021. URL : http://journals.openedition.org/entrelacs/6202 ; DOI : https:// doi.org/10.4000/entrelacs.6202

Ce document a été généré automatiquement le 15 décembre 2021.

Tous droits réservés 


\section{Filmographie de Laurent Roth}

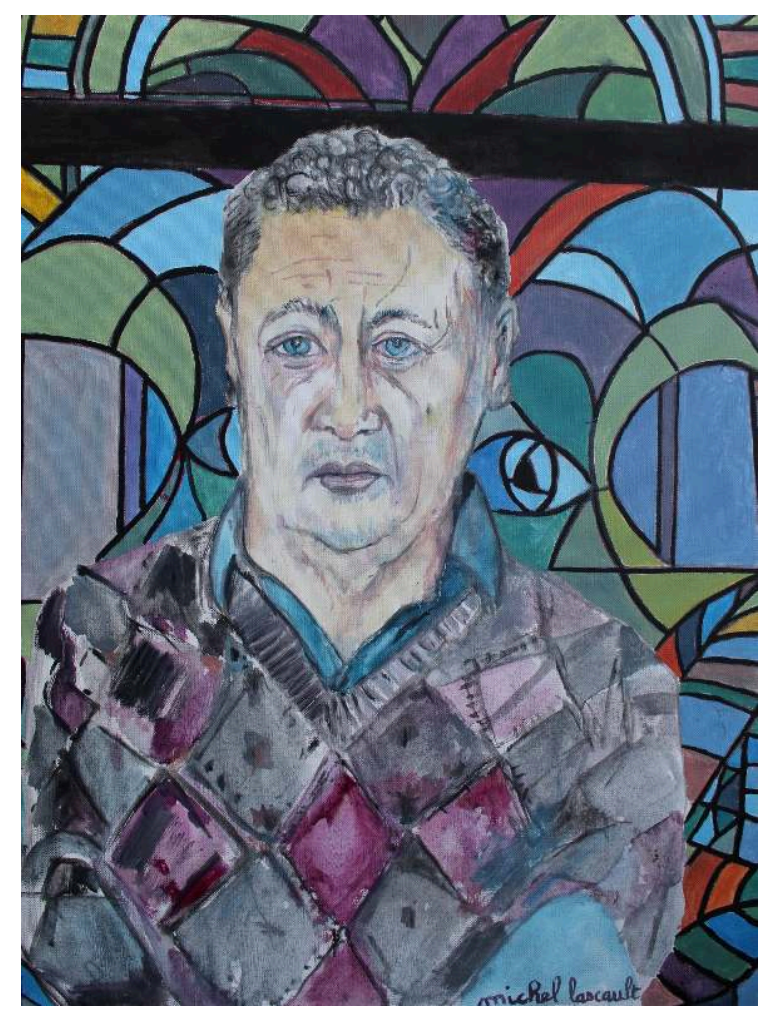

Laurent Roth. Portrait au vitrail.

Peinture de Michel Lascault, octobre 2021.

Marie ou le retour, 16mm, couleur et noir et blanc, 10' - 1984

Avec la participation de Roger-Philippe Bertozzi, Franco Cigana, Annette Coll, Daniel Mozis - Image : Sophie Maintigneux, Isabelle Czajka - Son : Emmanuel Maintigneux Montage: Alain Sanchis, Richard Beaudonnat - Musique: Mozart - Scénario et réalisation : Laurent Roth - Production : Edmée Millot, Archibald Films - Distribution : Laurent Roth. 
Joseph est parti à la guerre, il est officier de marine. Marie et son fils l'attendent dans une villa au bord de la mer. Un jardinier subvient à leurs besoins. Au fil des jours, sur ce petit théâtre de l'attente, chacun procède aux rites de la patience. Le retour, c'est le retour du père et le retour au père. Le fils rejoint le père. Il en a les yeux brûlés.

\section{Henri Alekan, des lumières et des hommes, 16mm, noir et blanc, 20 ' - 1986}

3 Image : Sophie Maintigneux, Isabelle Czajka - Son : Emmanuel Maintigneux - Montage : Alain Sanchis, Richard Beaudonnat - Musique: Charles-Henri-Valentin Alkan Entretien et réalisation : Laurent Roth - Production : Edmée Millot, Archibald Films Remastérisation 2012 : Paul Champart, Dominique Ciekala, Inthemood - Distribution: Laurent Roth.

4 Tourné en avril 1984 à Boulogne, cet entretien saisit Henri Alekan, chef-opérateur mythique de la "Qualité française ", au moment de son come-back dans le cinéma d'auteur (Robbe-Grillet, Ruiz, Wenders, Straub et Huillet, Gitaï) et de la parution de son livre somme: Des Lumières et des Ombres, véritable traité philosophique de l'éclairagisme. Rencontre avec un homme au sommet de son art.

\section{Les Yeux brûlés, 35 mm, couleur et noir et blanc, 59' - 1986}

5 Avec Mireille Perrier et la participation de Daniel Camus, Raoul Coutard, Raymond Depardon, Pierre Ferrari, Marc Flament, Pierre Schoendoerffer - Silhouette photographe et photos de plateau: Adjudant-chef Patrice George - Chef opérateur: Bernard Miale - Son : Jean-Paul Bigorgne - Montage : Marie-Christine Dijon- Mixage : Monique Wisniewski - Musique : J.-S. Bach ; Chant parachutiste traditionnel - Chargé de production : Philippe Viney - Conseiller militaire de réalisation : Commandant Alain Boitard - Conseiller technique : André Lebon - Scénario et réalisation : Laurent Roth Production: Établissement Photographique et Cinématographique des Armées Distribution : Shellac.

Une jeune femme vient chercher à l'aéroport de Roissy une cantine militaire qui lui est retournée. Il s'agit des effets et clichés de Jean Péraud, reporter photographe disparu à Dien Bien Phu le 8 mai 1954. Bientôt, la discussion s'engage entre la jeune femme et les anciens compagnons de presse de Péraud qui sont présents. À travers les souvenirs et récits qu'elle suscite, ressurgissent les questions toujours actuelles sur ce qui fait l'image de guerre : le reporter est-il témoin ou combattant ? Se protège-t-il des effets de la guerre en la filmant ou bien au contraire prend-il plus de risques pour rendre son témoignage ? Peut-on parler d'art devant cette image faite face à la mort?

\section{Ave Maria, tourné-monté, super 8 mm, 4' - 1987}

7 Musique: Antonio Ruiz-Pipo - Voix: Agritt - Conformation: Nicolas Gayraud Distribution : Laurent Roth.

8 Une mèche soufflée, une pierre d'angle rejetée, des pains multipliés, du blé fauché et la faucheuse qui nous regarde. Quelques versets bibliques illustrés par des plans volés dans la campagne durant l'été. 


\section{Modèle depuis toujours, vidéo Umatic, 10' - 1988}

Avec la participation de Claire-Marie Magen et Michel Lascault - Voix et texte : ClaireMarie Magen - Image, son : Laurent Roth - Montage, mixage : Martin Marini, Sylvain Huet - Musique : Beethoven - Scénario et réalisation: Laurent Roth - Production: Laurent Roth/Tutti Création/CFT Gobelins - Remastérisation 2015 : Paul Champart, Inthemood - Distribution : Laurent Roth.

10 Un modèle professionnel témoigne à partir de deux séances de pose, l'une collective, l'autre individuelle : Claire-Marie Magen dit ce qu'elle vit, craint, espère, ce qu'elle souhaite donner lorsqu'elle s'offre nue au regard de l'artiste. Claire-Marie Magen est filmée par Laurent Roth pour la première fois à la caméra : à l'Académie de la Grande Chaumière à Paris $6^{\text {ème }}$; et en privé et en plein air avec le peintre Michel Lascault à Mons-en-Montois (Seine-et-Marne).

\section{L'Impromptu de Jacques Copeau, vidéo Bétacam, 59' - 1993}

11 Avec Catherine Dasté, Marie Rivière, Olivier Cruveiller, Germain - Premier assistantréalisateur: Anne Moullahem - Image: Jacques Pamart - Son: Myriam René Electricien: Claude Pezet - Machiniste: Edouard Mazzochi - Costumière: Pascale Lavandier - Maquilleuse: Lucie Codomier - Montage: Bénédicte Mallet - Mixage: Nicolas Joly - Musique : Arvo Pärt - Scénario, adaptation, réalisation : Laurent Roth Production déléguée : Yvon Davis, Agat Films - Production: Agat Films, La Sept/Arte, l'INA, le Centre Georges Pompidou - Distribution : Doc \& Film International.

Jeu théâtral alternant avec des documents d'archives, cette fantaisie documentaire mélange les genres. Un studio plongé dans l'obscurité, un tréteau nu, quatre chaises, une table, un magnétophone, un projecteur, quelques livres et photos : voici les éléments de la machine à mémoire de l'Impromptu de Jacques Copeau. Sous l'impulsion discrète d'un régisseur replié dans les gradins (Laurent Roth lui-même), quatre comédiens s'emparent des textes de Copeau. Sur une autre scène, vont et viennent témoignages et documents d'archives qui sont comme autant d'indices servant à cerner la figure du réformateur du théâtre français. Enfin, la voix de Copeau qui enregistra ses souvenirs à la radio en 1945 , hante le cours du récit, jusqu'à l'apparition spectrale du « patron » à la fin du film.

\section{Ranger les photos, tourné-monté, mini DV, 14' - 1998 (coréalisation : Dominique Cabrera)}

13 Avec Dominique Cabrera - Image, son: Laurent Roth - Scénario, réalisation: Dominique Cabrera, Laurent Roth - Remastérisation 2009: Nicolas Djaal, Le Centquatre - Distribution : Ad Libitum.

«Avril 1998. Dominique Cabrera emménage la nouvelle maison qu'elle vient d'acheter à Montreuil. Je lui rends visite avec ma nouvelle caméra. Nous décidons après un bon repas de faire un film tous les deux. Nous nous fixons une règle : douze plans-séquence, en tourné-monté, avec fondu à l'ouverture et à la fermeture. Pas de montage donc; le film montré est le rushe tourné ce jour-là. Le sujet sera le rapport qu'entretient 
Dominique aux photos de famille, au cinéma, aux traces du bonheur. J'ai tourné ce film, je l'ai oublié, je le retrouve onze ans plus tard... » (Laurent Roth)

\section{Une maison de famille, DV cam, 37' - 2004}

15 Avec Laurent Roth - Image : Esti, Antoine Tracou - Image additionnelle : Jean-Charles Fitoussi - Son : Alberto Crespo, Patrick Allex - Montage : Ludovic Berrivin - Mixage : Jean-Marc Schick - Conformation, étalonnage : Romain Pierrat - Musique originale : Jean-Christophe Marti - Accordéon: Alain Dechaume - Musique additionnelle : J.-S. Bach - Texte, voix, scénario, réalisation: Laurent Roth - Production: Marie-Claude Reverdin, Cauri Films - Production: Cauri Films, France 2, Moulin d'Andé-Céci Distribution : Laurent Roth.

Version courte de J'ai quitté l'Aquitaine, qui n'inclut pas les «bobines de film de famille » dans la thérapie du patient...

\section{J'ai quitté l'Aquitaine, DV Cam, 52' - 2005}

Avec Laurent Roth - Image : Esti, Antoine Tracou - Image additionnelle : Jean-Charles Fitoussi - Son : Alberto Crespo, Patrick Allex - Montage : Ludovic Berrivin - Mixage : Isabelle Rougeot - Musique originale: Jean-Christophe Marti - Accordéon: Alain Dechaume - Musique additionnelle: J.-S. Bach - Texte, voix, scénario, réalisation: Laurent Roth - Production: Marie-Claude Reverdin, Cauri Films - Production : Cauri Films, France 3 Aquitaine, Moulin d'Andé-Céci - Distribution: ADAV Europe, Laurent Roth.

18 Le narrateur, interné dans un asile, tente de trouver la guérison en convoquant l'ensemble des membres de sa famille pour un test collectif : il s'agit, à l'aide d'une boîte de jeu de construction et de bobines de films de famille, d'essayer de restituer le plus exactement possible le bonheur tel qu'il était dans la maison de famille du Cap-Ferret, disparue il y a maintenant vingt-cinq ans. Mais rien ne va se passer comme prévu et tout se termine en chanson...

\section{La Chose, vidéo HD, 52' - 2010}

Captation de La Chose, une pièce de Laurent Roth - Avec Mathieu Amalric et Mireille Perrier - Mise en voix de l'auteur - Équipe captation : Arte studio - Cadreurs : Thierry Augé, Olivier Ziel, Jonas Bergot - Son : Hugues Petit - Réalisation : David Guedj - Chargé de production: Pascal Sottovia - Production: Théâtre du Rond-Point/Artelive web Distribution : Arte.

« Je suis un enfant de survivant. Dans ce texte, ni l'Holocauste ni la Shoah ne sont nommés. Je fais comme si la Chose n'avait pas eu lieu. Mais ça ne trompe personne. Il y a deux voix en moi : la voix de l'enfance intacte, qui ne veut rien savoir; et celle de la conscience déchirée devant le défaut supplicié du père. Entre ces deux voix, quelle voie pour devenir un sujet ? C'est en rencontrant beaucoup d'autres enfants de survivants que j'ai écrit ce texte. Ce monologue à deux voix est plein de leur voix singulière : grâce à eux, j'ai pu cesser d'être une exception. La Chose esquisse cette fragile invention du bonheur : où le vivre ne soit plus hanté par le survivre.» (Laurent Roth) 


\section{Écoute Israël, tourné-monté, vidéo DV Cam, 5' - 2014}

Avec Mathieu Amalric et Mireille Perrier - Image : Esti, Hugues Landry - Son, mixage : Dominique Ciekala - Montage : Ludovic Berrivin - Conformation, étalonnage, trucages : Paul Champart - Texte et réalisation : Laurent Roth - Production déléguée : Hugues Landry, Inthemood - Production : Inthemood, Théâtre du Rond-Point - Distribution: Laurent Roth.

En un plan séquence, à deux caméras, le poème de Laurent Roth lu par Mathieu Amalric au Théâtre du Rond-Point : la poésie, terre commune, terre d'accueil pour un possible dialogue israélo-palestinien.

\section{La Joie, DV Cam, 53' - 2015}

Avec Mathieu Amalric et Mireille Perrier - Image : Esti, Hugues Landry - Son, mixage : Dominique Ciekala - Montage : Ludovic Berrivin - Conformation, étalonnage, trucages : Paul Champart - Musique : Mozart - Texte et réalisation : Laurent Roth - Production déléguée: Hugues Landry, Inthemood - Production: Inthemood, Théâtre du RondPoint, Cinaps TV - Distribution : Magnolias Films.

Dans un avion entre Paris et Tel-Aviv. LUI accompagné par ELLE, transporte la dépouille de son père rescapé de la Shoah, en Israël. Mathieu Amalric et Mireille Perrier lisent le texte de La Joie de Laurent Roth au Théâtre du Rond-Point, dans un filmage somnambule et halluciné, où s'invitent comme autant de flashes et de blessures les images d'un voyage en Israël durant la première Intifada...

\section{Pierre Schœndoerffer, la peine des hommes, 58' - 2017}

Montage, étalonnage : Gaël Rougeron - Montage son, mixage : Frédéric Giménez Musique : J.-S. Bach - Chef du pôle Archives de l'Ecpad, production exécutive : Xavier Sené - Texte, voix, réalisation : Laurent Roth - Production:Ecpad - Distribution: Shellac.

Intégrale de l'entretien entre Pierre Schœndoerffer et Mireille Perrier lors du tournage des Yeux Brûlés, reconstitué d'après les rushes sonores du film et selon une méthode de restauration expérimentale et inédite : l'auteur de la 317ème Section et du Crabe Tambour $\mathrm{y}$ commente avec passion le métier de la guerre, l'art de la filmer, et le sort de son frère d'arme, Jean Péraud, disparu à Dien Bien Phu. Durant cet entretien, en parlant à une femme, jeune, et comédienne, on comprend très vite qu'il s'agit peut-être aussi d'une autre guerre pour Pierre Schœndoerffer: la guerre des mémoires, et la guerre des sexes... En conclusion, un montage d'archive présente la libération de Pierre Schoendoerffer au milieu des soldats français prisonniers du Vietminh durant l'été 1954.

\section{Raoul Coutard, J'ai pas une tête de mort, 30', 2017}

Montage, étalonnage : Gaël Rougeron - Montage son, mixage : Frédéric Giménez Musique : J.-S. Bach - Chef du pôle Archives de l'Ecpad, production exécutive : Xavier Sené - Texte, voix, réalisation : Laurent Roth - Production:Ecpad - Distribution: Shellac. 
Intégrale de l'entretien de Raoul Coutard avec Mireille Perrier lors du tournage des Yeux Brûlés, reconstitué d'après les rushes sonores du film et selon une méthode de restauration inédite : le chef-opérateur mythique de la Nouvelle vague y commente avec sobriété le métier de la guerre, l'art de la filmer, et le sort parfois tragique des reporters de guerre. Ce court-métrage présente de nombreux documents inédits : les photographies couleur des peuples Pnong en Indochine et les première images publiques de Raoul Coutard lorsqu'il était photographe officiel du général de Lattre de Tassigny.

\section{Le Pays fantôme, tourné-monté, 10', super 8 mm - 2019}

Son, montage: Nicolas Gayraud - Montage son, mixage: Jean-Marc Schick Conformation, étalonnage, générique : Romain Pierrat - Musique : Schumann - Piano : Joannès Roth - Texte, voix, scénario, réalisation : Laurent Roth - Production : Stéphane Jourdain, La Huit - Distribution : La Huit.

Bobine trouvée sur Ebay: un soldat filme de sa fenêtre la cour de sa caserne. Nous sommes dans les années soixante-dix et j'essaie d'imaginer ce qu'il peut bien avoir en tête.

\section{Amos Gitaï, la violence et l'histoire, video HD, 75' - 2020}

31 Avec Amos Gitaï et Laurent Roth - Image : Jérôme Colin, Felix Albert, Arthur Lormeau Prise de son, montage son, mixage: Laurent Thomas - Montage: Ginette Lavigne Musique originale : Joannès Roth, interprétée au piano par Pierre Fleury - Direction de production : Anne Louis - Producteur : Gérald Collas - Scénario, entretien, texte, voix et réalisation : Laurent Roth - Production INA, avec la participation de Ciné + et de France TELEVISIONS - Distribution : INA.

Un studio dans le noir, un grand écran plasma et deux regards : celui d'Amos Gitaï et celui de Laurent Roth, en dialogue autour des extraits d'une œuvre où la fiction et le documentaire sont en confrontation constante. Conçu comme une visite d'atelier, ce film met en œuvre une réflexion sur les débuts du cinéaste israélien, avec notamment la trilogie de La Maison (House), puis sur l'ensemble des œuvres dédiées à Yitzhak Rabin, auquel Gitaï a consacré inlassablement films, expositions, performances théâtrales, dénonçant les dérives sectaires de la société israélienne: son geste de mémoire est unique dans l'histoire du cinéma.

\section{Amos Gitai, Yitzhak Rabin, gestes de mémoire, video HD, 59' - 2020}

Avec Amos Gitaï et Laurent Roth - Image : Jérôme Colin, Felix Albert, Arthur Lormeau Prise de son, montage son, mixage : Laurent Thomas - Montage: Ginette Lavigne Musique originale : Joannès Roth, interprétée au piano par Pierre Fleury - Direction de production : Anne Louis - Producteur : Gérald Collas - Scénario, entretien, texte, voix et réalisation : Laurent Roth - Production INA, avec la participation de Ciné + et de France TELEVISIONS - Distribution : INA.

Un studio dans le noir, un grand écran plasma et deux regards : celui d'Amos Gitaï et celui de Laurent Roth, en dialogue autour des extraits d'une œuvre où la fiction et le documentaire sont en confrontation constante. 25 ans après l'assassinat du premier 
ministre israélien, ce film met en œuvre une réflexion sur l'ensemble des œuvres dédiées à Yitzhak Rabin, auquel Gitaï a consacré inlassablement films, expositions, performances théâtrales, dénonçant les dérives sectaires de la société israélienne : son geste de mémoire est unique dans l'histoire du cinéma.

Haïfa, la rouge, video HD, 15' - 2020

Avec Amos Gitaï et Laurent Roth - Image : Jérôme Colin, Felix Albert, Arthur Lormeau Prise de son, montage son, mixage: Laurent Thomas - Montage: Ginette Lavigne Musique originale : Joannès Roth - Direction de production: Anne Louis - Producteur : Gérald Collas - Scénario et réalisation : Laurent Roth - Production INA Distribution: INA.

Le monde d'Amos Gitaï en 15 minutes et 3 cartes de géographie de sa ville natale : les lieux où son père, le célèbre architecte Munio Gitaï Weinraub, a construit. Les lieux où il a grandi. Les lieux où il a tourné, depuis son documentaire Wadi en 1981.

\section{La Nymphe Scylla (Lettre à Jacky Evrard), tourné-monté, super 8mm,} $16^{\prime}-2021$

37 Son, montage : Nicolas Gayraud - Musique additionnelle : Jean-Marie Leclair - Texte, voix, scénario, réalisation : Laurent Roth - Distribution : Laurent Roth.

"A l'occasion du confinement, je retrouve les rushes de mon premier film, tourné avec mes profs de lycée. Je voulais adapter Les Métamorphoses d'Ovide pour évoquer la nymphe Scylla poursuivie par la vengeance de la magicienne Circé... Cet apprentissage du cinéma réserve bien des surprises! » (Laurent Roth) 